\title{
Molecular detection and antimicrobial resistance of Clostridium perfringens isolated from diabetic patients and bullet wounds
}

\author{
Aliaa Mohamed Hmood, Maysa S M Al-Shukri, Alaa H Al-Charrakh* \\ Department of Microbiology, College of Medicine, Babylon University, Hillah, Iraq
}

ARTICLE INFO

Article history:

Received on: March 28, 2018

Accepted on: April 27, 2018

Available online: January 20, 2019

\section{Key words:}

Molecular detection,

Internal transcribed spacer gene,

Antibiotic susceptibility,

Clostridium perfringens,

Diabetic patients

\begin{abstract}
Clostridium perfringens is a major cause of gas gangrene. The morbidity of $C$. perfringens is connected with producing toxins. This cross-sectional study was designed to isolate, genetically diagnose, and study the antibiotic susceptibility patterns of C. perfringens isolated from clinical samples. Different wound swabs (from diabetic patients, cellulitis, and bullet wounds) were taken from 140 patients. For isolation of anaerobic bacteria, samples (in thioglycolate broth) were immediately incubated anaerobically then identified according to the cultural properties and biochemical tests. DNA was extracted from all specimens. Polymerase chain reaction was applied for detection of $16 \operatorname{SrRNA}$ and internal transcribed spacer (ITS) genes of $C$. perfringens. The susceptibility of bacterial isolates to different antibiotics was determined using Vitek 2 system and disk diffusion test. Out of 140 clinical samples collected during this study, $3(2.14 \%)$ C. perfringens isolates were recovered of which 2 isolates $(1.43 \%)$ obtained from diabetic patients and one $(0.71 \%)$ from bullet wounds. Results also showed that only 7 isolates $(5 \%)$ were detected by a molecular method using specific primers 16S rRNA and ITS genes of $C$. perfringens. Results of antibiotic susceptibility testing showed that all isolates were highly susceptible to penicillins and $\beta$-lactamase inhibitors, metronidazole, and aminoglycosides. On the other hand, all isolates were highly resistant to tetracycline, levofloxacin, and erythromycin. The susceptibility patterns of $C$. perfringens isolates showed that all isolates were multidrug resistance. Using the amplification of ITS gene increases specificity and sensitivity (by reducing non-specific annealing and primer dimer formation) which increases the probability of detection of suspected C. perfringens isolates.
\end{abstract}

\section{INTRODUCTION}

Clostridium is a main cause of gas gangrene. It is a disease, related to wound infection, with possibly fatal prognosis in the situation of treatment's delays. In the lack of early essential surgery, antibiotic therapy and (if accessible) hyperbaric treatment result in the extent of toxins in the body causing shock, coma, and death. Due to the force of formed toxins, this pathogen is a key pillar for the manufacture of biological weapons. It can possibly be used to make outbreaks of food poisoning and by missiles contamination by spore, result in increased morbidity of gas gangrene in injured soldiers [1].

Traditionally in the past, the identification and taxonomy of eubacteria are grounded on phenotypic appearances. Different molecular procedures are employed in bacterial classification that is sensitive and specific methods for the recognition and identification

\footnotetext{
*Corresponding Author:

Alaa H Al-Charrakh,

Department of Microbiology,

College of Medicine,

Babylon University,

Hillah, Iraq.

Email: ahani67@gmail.com
}

of microorganisms which include hybridization, polymerase chain reaction (PCR), real-time PCR, and microarray [2].

Many scientists have employed rRNA genes for identification, characterization, and categorization of members of the genus Clostridium and as a taxonomic tool in the investigations of relationships with other pathogenic and nonpathogenic bacteria. This is done by nucleotide sequence analysis of genes in the rRNA operon, as the $16 \mathrm{~S}$ and $23 \mathrm{~S}$ rRNA genes, by the use of genus- and species-specific regions of $16 \mathrm{~S}$ rRNA hybridize with DNA probes for bacterial species identification [3].

The growing resistance to antibiotics among anaerobic pathogens has been an international problem in the past two decades. The degrees of resistance may display clinically significant differences among geographic parts and among countries [4].

Resistance to tetracycline (defined as minimum inhibitory concentration [MIC] $>2 \mu \mathrm{g} / \mathrm{mL}$ ) has been recognized in up to $75 \%$ of C. perfringens isolates recovered from commercial poultry, but there are few records in humans. The activities of tetracycline against other clostridial species in general have not been well documented [5].

Clindamycin has an extensive range of activity against anaerobic bacteria and has established its efficacy in clinical tests [6]. It belongs 
to the macrolide, lincosamide, and streptogramin family that acts through inhibition of protein synthesis. Bacterial resistance to this family may be stated through different mechanisms including target site modification, enzymatic antibiotic inactivation, and macrolide efflux pump [7].

The aim of this study was to isolate, genetically diagnose, and study the antibiotic susceptibility patterns of $C$. perfringens isolated from clinical samples.

\section{MATERIALS AND METHODS}

\subsection{Study Design and Bacterial Isolates}

This cross-sectional study was planned to assess the occurrence of $C$. perfringens isolates obtained from clinical specimens and to detect their antibiotic susceptibility patterns. At the beginning of this study, 140 different clinical samples were collected during the period of February-October 2016 from the main two hospitals in Babylon province/Iraq.

\subsection{Ethical Approval}

Verbal consent was taken from each patient before sampling. Investigative standards were strictly preserved, chiefly concerning confidentiality. Besides, this study was unrevealed, the participation of patients was optional, and verbal consent was obtained before data uptake process was started.

\subsection{Samples Collection}

Different wound swabs were collected from patients admitted to the outpatient clinics in two hospitals of Babylon province, Iraq; medical Marjan city and Hillah general teaching hospital. The study involved 140 patients subjected to sampling which included wound swabs from diabetic patients (no $=78$ ), cellulitis (no $=25$ ), and bullet wounds $($ no $=37)$. The samples were taken from both male and female patients with age ranged from 30 to 75 years. The patients within 3 days of antibiotic treatment and those with cleaned and sterilized wounds were excluded from sampling.

The specimens were collected in proper ways to avoid any possible contamination. For microbiological sampling, the area to be sampled was isolated with sterile cotton swabs. After obtaining the permission from the subjects for examination and sampling, and before cleaning the wound or sterilizing, two swabs were taken for each sample; one for direct DNA extraction and the other for culturing. Then swabs were rotated carefully, and samples were taken by inserting swabs deeply in the wound site, and finally, they were put in a plain tube containing $1 \mathrm{~mL}$ of thioglycolate broth transport medium. The specimen was transported to the laboratory using iced-box.

Samples obtained from the wound sites swabs were placed in tube with thioglycolate broth at $37^{\circ} \mathrm{C}$ for $24 \mathrm{~h}$ in anaerobic incubator then cultured on blood agar plates and selective media immediately and finally incubated anaerobically in an anaerobic jar at $37^{\circ} \mathrm{C}$ for $48 \mathrm{~h}$ plus $10 \% \mathrm{CO}_{2}$. Isolates were subjected to identification according to the cultural properties, microscopic examination, and biochemical tests [8].

\subsection{Isolation and Identification of $\boldsymbol{C}$. perfringens}

The presumptive identification of isolated bacteria was carried out by Gram staining and cultural characteristics of bacteria in special media such as thioglycolate broth, blood agar, Columbia agar, and Iron milk medium.
The completed tests of the isolated culture were done by lecithinase activity of bacteria in lactose-egg yolk agar, indole test, catalase test, and fermentation of various carbohydrates. A single colony was taken from each primary positive culture. Its identification depended on morphology properties (colony size, shape, color, translucency, edge, and elevation of texture). The colonies were then investigated by Gram stain to observe bacterial cells. Specific biochemical tests were done to reach the final identification.

\subsection{Genotypic Detection of $\boldsymbol{C}$. perfringens isolates}

PCR was used for amplification of specific genes belonged to suspected $C$. perfringens isolates. Two pairs of primers were employed to increase the probability of detection. The first was 16SrRNA gene, while the second target was the more conserved regions of the internal transcribed spacer (ITS), by the usage of touch down protocol PCR which used to increase specificity and sensitivity, reducing nonspecific annealing, and primer dimer formation.

The primer sequence of these genes was (16SrRNA F: CGAGTCGGCATCTAATACTAT, 16SrRNA R: AAAACGACATTTCTGCCGC) $279 \mathrm{bp}$; the conditions were $94^{\circ} \mathrm{C}$ $2 \min \times 1,94^{\circ} \mathrm{C} 1 \mathrm{~min}, 53^{\circ} \mathrm{C} 1 \mathrm{~min} \times 35,72^{\circ} \mathrm{C}, 1 \mathrm{~min}$, and $72^{\circ} \mathrm{C} 5 \mathrm{~min}$ $\times 1$ [9]. For (ITS F: GGCGTATTTGGGCATCCGAGTAACG, ITS R: TCAAATCCAGCGGGTATTAACCGCCT) $500 \mathrm{bp}$, the conditions were $95^{\circ} \mathrm{C} 5 \mathrm{~min} \times 1,94^{\circ} \mathrm{C} 30 \mathrm{~s}, 45^{\circ} \mathrm{C} 30 \mathrm{~s}, 72^{\circ} \mathrm{C} 30 \mathrm{~s}$, and $72^{\circ} \mathrm{C} 1 \mathrm{~min} \times 30$ [10].

\subsection{Antimicrobial Susceptibility Testing}

The susceptibility of bacterial isolates to different antibiotics was determined using Vitek 2 system (BioMérieux, France) and disc diffusion method (DDT). The results were interpreted according to CLSI guidelines [11]. In Vitek 2 system, kit for GP (Code 580) was used for determination of MICs of bacterial isolates.

The definition of antibiotic resistance patterns was determined according to Magiorakos et al. [12]. Multidrug resistance (MDR) was defined as the phenotype of non-susceptibility to representative antimicrobial agents of at least three different classes of drugs.

\section{RESULTS AND DISCUSSION}

\subsection{Isolation and Identification of $\boldsymbol{C}$. perfringens Isolates}

A total of 140 clinical samples were collected during this study, $3(2.14 \%)$ C. perfringens isolates were recovered, of which 2 isolates $(1.43 \%)$ were obtained from diabetic patients, and 1 isolate $(0.71 \%)$ was obtained from bullet wounds. No isolates were recovered from the other clinical samples (cellulitis) [Table 1]. The majority of C. perfringens isolates were detected in diabetic patients and bullet wounds in $1.43 \%$ and $0.71 \%$, respectively.

The identification of $C$. perfringens isolates depends mainly on the cultural and biochemical characteristics and also microscopic patterns. The result demonstrated that $C$. perfringens characteristically is a large, boxcar-shaped, Gram-positive rod sub-terminal spore, and after cultured in anaerobe blood agar plates under anaerobic conditions at $37^{\circ} \mathrm{C}$, creamy yellow to gray colonies are formed within $48 \mathrm{~h}$ in $10 \% \mathrm{CO}_{2}$. The colony margins can be smooth, giving the appearance of a round, a little-raised colony, or rough, in which instance the colonies are moderately flat.

On egg yolk agar, isolates showed high lecithinase activity which indicated by opaqueness and halo around the colonies due to the breakdown of lecithin in the egg yolk. Biochemical identification 
Hmood, et al.: Journal of Applied Biology \& Biotechnology 2019;7(01):54-59

Table 1: Numbers of C. perfringens isolates detected by culture and PCR recovered from different sources of infections

\begin{tabular}{lccc} 
Source of wound swabs & Number of samples & Number of isolates by culture (\%) & Number of isolates by PCR (ITS* and 16SrRNA) (\%) \\
Diabetic patients & 78 & $2(1.43)$ & $5(3.57)$ \\
Bullet wounds & 37 & $1(0.71)$ & $2(1.42)$ \\
Cellulitis & 25 & $0(0.00)$ & $0(0.00)$ \\
Total & 140 & $3(2.14)$ & $7(5)$ \\
\hline
\end{tabular}

*ITS: Internal transcribed spacer. C. perfringens: Clostridium perfringens, PCR: polymerase chain reaction

showed gas and acid production from fermented glucose, sucrose, and lactose. Motility test was done for all isolates by the use of motility media and compared with motile bacteria as a positive control. On Ironmilk presumptive test, the reaction characterized by fast coagulation of milk followed by the breaking of curd into a spongy mass which usually rises above the medium surface. In microscopic examination, the organisms were small rod-shaped appearance, arranged in single or pair, catalase, and indole negative.

The present study was focused on C. perfringens as the term clostridial myonecrosis better defines both the causative agent and the target tissue. Earlier to the advent of antibiotics and mobile army surgical hospitals, as many as $5 \%$ of battlefield injuries were complicated by this state. Currently, $90 \%$ of contaminated wounds reveal clostridial organisms, but $<2 \%$ develop clostridial myonecrosis. This underscores the significance of host and local wound factors in the progress of this process, rather than the occurrence of the bacteria in the wound [13]. Another study was done by Méndez et al. [14] found that $C$. perfringens was the well-known cause of clostridial gas gangrene in $80-90 \%$ of cases.

The low number of $C$. perfringens isolates recovered in this study may be due to several reasons. First: These bacteria are strict anaerobe, second the lack of distinction between contamination and infection, and third the misunderstanding between gas gangrene and several clostridial infections, and other bacterial and nonbacterial injuries promoting gas gangrene [15].

The rate of isolation was detected in diabetic patients $(1.43 \%)$ as shown in Table 1. As diabetes mellitus is increasing in incidence and now presents one of the chief health problems worldwide.

This result was compatible with Rechner et al. [16] who found that $C$. perfringens was linked to extremely serious soft-tissue and muscle infections, such as gas gangrene, which often happened in patients who had fecally contaminated farm wounds and war wounds.

However, in the previous study done by Wang et al. [17], C. perfringens had been detected in the majority of patients with gas gangrene. Low sensitivities of culturing techniques and sampling of a limited number of wound sites may have caused an underestimate of the true prevalence of this organism in these studies. In some cases, it may be triggered by Group A Streptococcus bacteria. The infection arises suddenly and spreads rapidly; it was usually developed at a recent surgery or injury site. In rare cases, it may occur spontaneously, without an actual cause [17].

C. perfringens is the most common cause of trauma accompanying gas gangrene with very high mortality rates [18]. As shown in Table 1, C. perfringens isolates were not detected in cellulitis swab samples. This may be due to small numbers of collected samples or due to the fact that cellulitis specifically affects the dermis and subcutaneous area, which may lead to the absence of any growth of anaerobic bacteria such as $C$. perfringens in the site of infection. In cellulitis the most bacteria commonly involved are streptococci and $S$. aureus [19].
Several studies determined that $C$. perfringens is the most common cause of clostridial cellulitis, which is frequently related to local trauma or recent surgery. Infection is less systemic than in clostridial myonecrosis, with localized infection and associated skin and soft tissue necrosis, but sparing of the fascia and deep muscles [1].

\subsection{Molecular Identification of $C$. perfringens by PCR Using Specific Primers}

As shown in Table 1, 7 isolates of $C$. perfringens of 140 were detected by a molecular method using specific primers $16 \mathrm{~S}$ rRNA, 16S-23S spacer rRNA (ITS) genes of $C$. perfringens.

Results of conventional PCR (for the amplification of specific 16SrRNA primer) revealed that of 140 samples, only 7 C. perfringens isolates $(5 \%)$ produced the specific 279 bp DNA fragment when compared with allelic ladder [Figure 1]. To confirm the previous result, both specific primers were used, genus- and species-specific ITS. This result showed an equal percentage of identification to $7 / 140(5 \%)$, produced the specific 500 bp DNA fragment when compared with allelic ladder [Figure 2].

The molecular markers used for bacterial identification are the species-specific 16S and 16S-23S spacer rRNA genes (ITS) for the detection of $C$. perfringens DNA, as both gave a close result. This method is a practical and useful method that could be easily employed. As these ribosomal genes are suitable molecular targets for bacterial classification, these genes are universal among bacteria and are conserved.

Detection of 16S rRNA gene (using specific primers corresponding to the this gene) permits for the molecular genotyping of C.perfringens [20].

Organism typing is achieved by culture filtrates with type-specific antisera; these are hard to find, very expensive, time-consuming, and ethically undesirable for tests in experimental animals. The usage of PCR can circumvent these problems and may be used to separate C. perfringens into its five toxin types [21].

\subsection{Antibiotic Susceptibility Testing}

Results of susceptibility testing using Vitek 2 system and DDT of $C$. perfringens isolates $($ no $=3$ ) against 24 antibiotics showed that all isolates were highly susceptible to penicillins (such as benzylpenicillin, piperacillin, and amoxicillin), and they were sensitive to $\beta$-lactam- $\beta$-lactamase inhibitor combinations, such as ampicillinsulbactam [Table 2]. They were also susceptible to metronidazole and aminoglycosides in 3/3. Furthermore, all isolates were highly resistant to tetracycline, levofloxacin, and erythromycin. However, these isolates expressed different degrees of susceptibility toward other antibiotics (linezolid, clindamycin, and imipenem) [Table 2].

The susceptibility of $C$. perfringens to penicillins (benzylpenicillin, piperacillin, and amoxicillin) was reported by several authors $[5,7]$. 


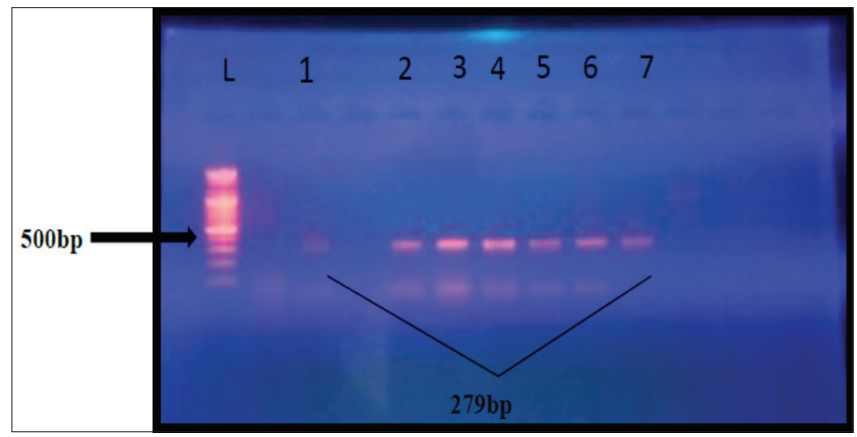

Figure 1: 1.5\% agarose gel electrophoresis at 70 volt for $50 \mathrm{~min}$ for Clostridium perfringens $16 \mathrm{SrRNA}$ polymerase chain reaction products visualized under ultraviolet light at $280 \mathrm{~nm}$ after staining with ethidium bromide. L: 1500 bp ladder; lane (1, 2, 3, 4, 5, 6, and 7) are positive. The amplicon size is $279 \mathrm{bp}$

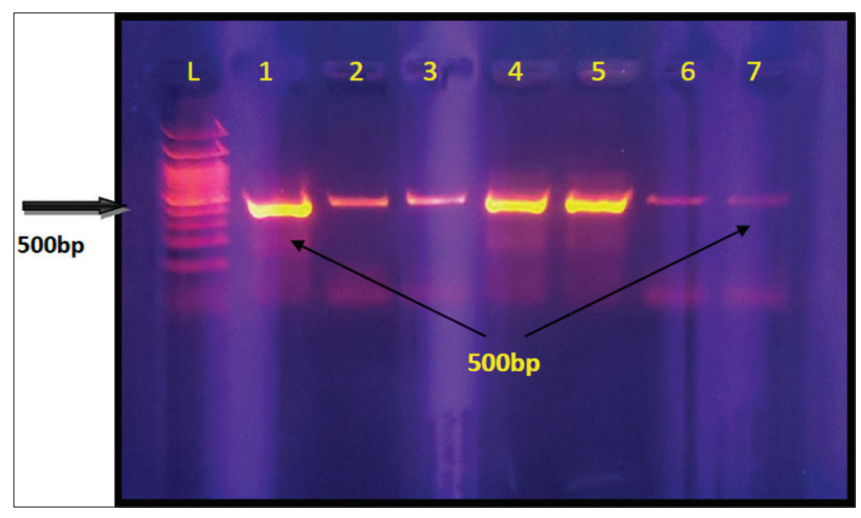

Figure 2: 1.5\% agarose gel electrophoresis at 70 volt for $50 \mathrm{~min}$ for Clostridium perfringens 16S-23S spacer rRNA polymerase chain reaction products visualized under ultraviolet light at $280 \mathrm{~nm}$ after staining with ethidium bromide. L: 1500 bp ladder; lane (1, 2, 3, 4, 5, 6, and 7) are positive. The amplicon size is $500 \mathrm{bp}$

Stevens et al. [5] found that beta-lactam antibiotics that interfere with cell wall biosynthesis during active growth, causing bactericidal activity against sensitive bacteria.

Clostridium perfringens, in addition to most other Clostridium species are normally, but not entirely susceptible to penicillin-G, amoxicillin, ticarcillin, piperacillin, imipenem, and the combinations of penicillins and beta-lactamase inhibitors [7].

Aggelidakis et al. [4] demonstrated that ampicillin-sulbactam or piperacillin-tazobactam or ticarcillin-clavulanate in combination with clindamycin or metronidazole are recommended empiric regimens, while antibiotic treatment should be designed according to the susceptibility results.

Other studies had been demonstrated that resistance to ampicillinsulbactam, amoxicillin is very rare. A low degree of resistance to ampicillin has been reported in several studies [7].

Results also found that all isolates were highly resistant to tetracycline, levofloxacin, and erythromycin [Table 2]. The susceptibility of C. perfringens to tetracyclines and erythromycin was reported by several authors [5]. Tansuphasiri et al. [22] found that $C$. perfringens isolated from human source showed the highest rate of resistance to tetracycline $(56.2 \%)$, followed by imipenem (24.9\%). Janvilisri et al. [23] found that tetracyclines were potent against $C$. perfringens which causes gas gangrene, the resistance to tetracyclines was relatively high $(41 \%)$. However, the highest resistance to tetracycline in $C$. perfringens isolates from human sources in antibiotics belonging to the groups of macrolides, and quinolones were not quite effective against $C$. perfringens isolates.

The $C$. perfringens isolates from this study were more susceptible to metronidazole and moxifloxacin in $3 / 3$. Highly sensitivity was also noted in aminoglycosides such as gentamycin (3/3) and Tobramycin $(2 / 3)$.

The use of metronidazole in the treatment of $C$. perfringens infections was reported by many authors [23,24]. Stevens et al. [24] demonstrated that the drugs were more active in the management of clostridial infection, and metronidazole and rifampicin were more effective in the treatment of fulminate gas gangrene caused by $C$. perfringens. Altın et al. [25] found that metronidazole has identical in vitro effectiveness against anaerobic bacteria and it has exceptional in vitro activity against most strict anaerobic bacteria, such as clostridia, Bacteroides fragilis group, and other species of Bacteroides, and fusobacteria.

The susceptibility of $C$. perfringens to aminoglycosides was reported by many studies [26,27]. Mingeot-Leclercq et al. [26] demonstrated that aminoglycosides are most effective against anaerobic bacteria. However, recent studies found that aminoglycoside antibiotics are used for Gram-negative bacterial coverage. Kitterer et al. [27] found that these antibiotics are frequently used in combination with an agent with activity against Gram-positive bacteria and one that covers anaerobes.

Results also found that all isolates were sensitive to vancomycin, teicoplanin, trimethoprim/sulfamethoxazole, and rifampicin [Table 2]. The susceptibility of $C$. perfringens to vancomycin, trimethoprim/ sulfamethoxazole and rifampicin was reported by many studies [18,24] which demonstrated that rifampicin was more effective in the treatment of clostridial infection. This drug was more efficacious in the management of gas gangrene caused by $C$. perfringens. Stevens et al. [18] demonstrated that definite treatment for post-traumatic gas gangrene due to $C$. perfringens should consist of vancomycin $(1 \mathrm{~g})$ since this species may be resistant to clindamycin.

Results of susceptibility patterns of $C$. perfringens isolates showed that all isolates were MDR. However, no isolate showed extensively drug-resistant or pandrug-resistant pattern of resistance [Table 3]. Akhi et al. [28] showed a moderate prevalence of antimicrobialresistant C. perfringens isolates. Among 56.95\% of antimicrobialresistant strains, $22.78 \%$ were single drug-resistant, and $34.17 \%$ were MDR. Gamboa-Coronado et al. [29] demonstrated that multi-resistance was detected in $C$. perfringens strains from human origin $(40 \%)$. They found that antibiotic resistance to at least one antibiotic was identified in $44 \%$ was resistant to tetracycline, $41 \%$ to clindamycin, $25 \%$ to chloramphenicol, $22 \%$ to penicillin, and $20 \%$ to metronidazole.

\section{CONCLUSION}

Using the amplification of ITS gene increases specificity and sensitivity (by reducing non-specific annealing and primer dimer formation) which increases the probability of detection of suspected $C$. perfringens isolates. The low number of $C$. perfringens isolates recovered in this study may be due to several reasons. First: These 
Table 2: Antibiotic resistance patterns of C. perfringens isolates using Vitek 2 system and DDT

\begin{tabular}{|c|c|c|c|c|c|c|c|c|c|c|c|c|c|c|c|c|c|c|c|c|c|c|}
\hline \multirow[t]{2}{*}{ Isolate } & \multicolumn{22}{|c|}{ Antibiotic } \\
\hline & $\mathbf{P}$ & PI & OXA & AMC & AMS & IPM & TE & TGC & MOF & LEF & CI & MTR & $\mathrm{CN}$ & TM & VA & TEC & $\mathbf{R A}$ & SXT & LNZ & $\mathbf{E}$ & FT & FA \\
\hline S1 & $\mathrm{S}$ & $\mathrm{R}$ & $\mathrm{S}$ & S & S & $\mathrm{R}$ & $\mathrm{R}$ & S & S & $\mathrm{R}$ & $\mathrm{R}$ & S & S & $\mathrm{S}$ & $\mathrm{S}$ & S & $\mathrm{S}$ & S & $\mathrm{R}$ & $\mathrm{R}$ & S & $\mathrm{R}$ \\
\hline S3 & $\mathrm{S}$ & $\mathrm{S}$ & $\mathrm{S}$ & $\mathrm{S}$ & $\mathrm{S}$ & $\mathrm{S}$ & $\mathrm{R}$ & $\mathrm{S}$ & $\mathrm{S}$ & $\mathrm{R}$ & $\mathrm{S}$ & $\mathrm{S}$ & $\mathrm{S}$ & $\mathrm{S}$ & $\mathrm{S}$ & $\mathrm{S}$ & $\mathrm{S}$ & $\mathrm{S}$ & $\mathrm{R}$ & $\mathrm{R}$ & $\mathrm{S}$ & $\mathrm{S}$ \\
\hline
\end{tabular}

P: Penicillin, OXA: Oxacillin, CN: Gentamicin, TM: Tobramycin, RA: Rifampicin, SXT: Trimethoprim-sulfamethoxazole, VA: Vancomycin, CI: Clindamycin, LNZ: Linezolid, TGC: Tigecycline, TE: Tetracycline, TEC: Teicoplanin, LEF: Levofloxacin, FT: Nitrofurantoin, MOF: Moxifloxacin, AMC: Amoxicillin, AMS: Ampicillin/sulbactam, IPM: Imipenem, PI: Piperacillin, MTR: Metronidazole, FA: Fusidic acid, E: Erythromycin. C. perfringens: Clostridium perfringens, DDT: Disc diffusion method

Table 3: Antibiotics resistance pattern of $C$. perfringens isolates

\begin{tabular}{ll} 
C. perfringens isolate & Antibiotic resistance pattern \\
S1 & $\mathrm{PI}^{\mathrm{r}}, \mathrm{IPM}^{\mathrm{r}} \mathrm{Tet}^{\mathrm{r}}, \mathrm{LEF}^{\mathrm{r}}, \mathrm{CI}^{\mathrm{r}}, \mathrm{LNZ}^{\mathrm{r}}, \mathrm{E}^{\mathrm{r}}, \mathrm{FA}^{\mathrm{r}}$ \\
$\mathrm{S} 2$ & $\mathrm{IPM}^{\mathrm{r}}, \mathrm{TE}^{\mathrm{r}}, \mathrm{LEF}^{\mathrm{r}}, \mathrm{TGC}^{\mathrm{r}}, \mathrm{CI}^{\mathrm{r}}, \mathrm{TM}^{\mathrm{r}}, \mathrm{LNZ}^{\mathrm{r}}, \mathrm{E}^{\mathrm{r}}, \mathrm{FT}^{\mathrm{r}}$ \\
$\mathrm{S} 3$ & $\mathrm{TE}^{\mathrm{r}}, \mathrm{LEF}^{\mathrm{r}}, \mathrm{LNZ}^{\mathrm{r}}, \mathrm{E}^{\mathrm{r}}$ \\
\hline
\end{tabular}

C. perfringens: Clostridium perfringens

bacteria are obligate anaerobe, second: The lack of separation between contamination and infection, and third: The misunderstanding between gas gangrene and various clostridial infections, and other bacterial and nonbacterial lesions simulating gas gangrene. The susceptibility patterns of $C$. perfringens found that all strains were extremely resistant to tetracycline, levofloxacin, and erythromycin but they were susceptible to vancomycin, teicoplanin, trimethoprim/ sulfamethoxazole, and rifampicin. However, all isolates showed MDR pattern of resistance.

\section{REFERENCES}

1. Claeys LG, Matamoros R. Anaerobic cellulitis as the result of Clostridium perfringens: A rare cause of vascular access graft infection. J Vasc Surg 2002;35:1287-8.

2. Ott SJ, Musfeldt M, Ullmann U, Hampe J, Schreiber S. Quantification of intestinal bacterial populations by real-time PCR with a universal primer set and minor groove binder probes: A global approach to the enteric flora. J Clin Microbiol 2004;422:566-72.

3. Wang Y, Qian PY. Conservative fragments in bacterial 16S rRNA genes and primer design for $16 \mathrm{~S}$ ribosomal DNA amplicons in metagenomic studies. PLoS One 2009;4:e7401.

4. Aggelidakis J, Lasithiotakis K, Topalidou A, Koutroumpas J, Kouvidis G, Katonis P. Limb salvage after gas gangrene: A case report and review of literature. World J Emerg Surg 2011;17:28.

5. Stevens DL, Aldape MJ, Bryant AE. Necrotizing fasciitis, gas gangrene, myositis, and myonecrosis. In: Cohen J, Powderly WG, Opal SM, editor. Infectious Diseases. Vol. 44. China: Mosbey Elsevier Inc.; 2010. p. 119-27.

6. Brook I, Wexler HM, Goldstein EJ. Antianaerobic antimicrobials: Spectrum and susceptibility testing. Clin Microbiol Rev 2013;26:526-46.

7. Roberts SA, Shore KP, Paviour SD, Holland D, Morris AJ. Antimicrobial susceptibility of anaerobic bacteria in New Zealand: 1999-2003. J Antimicrob Chemother 2006;57:992-8.

8. Takazawa K, Otsuka H, Nakagawa Y, Inokuchi S. Clinical features of non-clostridial gas gangrene and risk factors for in-hospital mortality. Tokai J Exp Clin Med 2015;40:124-9.

9. Rönner SG, Stackebrandt E. Identification of Clostridium perfringens by $16 \mathrm{~S}$ and $23 \mathrm{~S}$ rRNA Oligonucleotide Probes. Syst App Microbiol 1994; 17:425-32.

10. Barry T, Colleran G, Glennon M, Dunican LK, Gannonl F.The $16 \mathrm{~s} / 23 \mathrm{~s}$ ribosomal spacer region as a target for DNA probes to identify eubacteria. PCR Methods Appl 2015;51:434-67.

11. Clinical and Laboratory Standards Institute. Performance Standards or Antimicrobial Susceptibility Testing; Twenty-Fifth Informational Supplement. Wayne, PA: CLSI document M100-S25. Clinical and Laboratory Standards Institute; 2015.

12. Magiorakos A, Srinivasan P, Carey RB, Carmeli Y, Falagas ME, Giske CG, et al. Multidrug-resistant, extensively drug-resistant and pan drug resistant bacteria: An international expert proposal for interim standard definitions for acquired resistance. Clin Microb Infect 2012;18:268-81.

13. Javed S, Rafeeq M, Tariq MM, Awan MA, Rashid N, Ali M. Study on in vitro biochemical growth characterization and assessment of hemolytic toxin of C. Perfringens type B and D. Pak J Zool 2012;44:1575-80.

14. Méndez MB, Goñi A, Ramirez W, Grau RR. Sugar inhibits the production of the toxins that trigger clostridial gas gangrene. Microb Pathog 2012;52:85-91.

15. Hausmann R, Albert F, Geissdorfer W, Betz P. Clostridium fallax associated with sudden death in a 16 year-old boy. J Med Microbiol 2004;53:581-83.

16. Rechner PM, Agger WA, Mruz K, Cogbill TH. Clinical features of clostridial bacteremia: A review from a rural area. Clin Infect Dis 2001;33:349-53.

17. Wang Y, Lu B, Hao P, Yan MN, Dai KR. Comprehensive treatment for gas gangrene of the limbs in earthquakes. Chin Med J (Engl) 2013;126:3833-9.

18. Stevens DL, Bisno AL, Chambers HF, Everett ED, Dellinger P, Goldstein EJ, et al. Practice guidelines for the diagnosis and management of skin and soft-tissue infections. Clin Infect Dis 2005;41:1373-406.

19. Vary JC, O’Connor KM. Common dermatologic conditions. Med Clin North Am 2014;98:445-85.

20. Wu J, Zhang W, Xie B, Wu M, Tong X, Kalpoe J, et al. Detection and toxin typing of clostridium perfringens in formalin-fixed, paraffin-embedded tissue samples by PCR. J Clin Microbiol 2009;47:807-10.

21. Piatti RM, Ikuno AA, Baldassi L. Detection of bovine clostridium perfringens by polymerase chain reaction. J Venom Anim Toxins Incl Trop Dis 2004;10:1678-99.

22. Tansuphasiri U, Matra W, Sangsuk L. Antimicrobial resistance among clostridium perfringens isolated from various sources in Thailand. Southeast Asian J Trop Med Public Health 2005;36:954-61.

23. Janvilisri T, Scaria J, Gleed R, Fubini S, Bonkosky MM, Gröhn YT, et al. Development of a microarray for identification of pathogenic clostridium spp. Diagn Microbiol Infect Dis 2010;66:140-7.

24. Stevens DL, Aldape MJ, Bryant AE. Life-threatening clostridial infections. Anaerobe 2012;18:254-9.

25. Altın C, Kanyılmaz S, Baysal S, Özin B. QT interval prolongation due to metronidazole administration. Anadolu Kardiyol Derg 2011;11:468-9.

26. Mingeot-Leclercq MP, Glupczynski Y, Tulkens PM. Aminoglycosides: Activity and resistance. Antimicrob Agents Chemother 1999;43:727-37. 
Hmood, et al.: Characterization of C. perfringens from clinical samples 2019;7(01):54-59

27. Kitterer D, Braun N, Jehs MC, Schulte B, Alscher MD, Latus J, et al. Gas gangrene caused by clostridium perfringens involving the liver, spleen, and heart in a man 20 years after an orthotopic liver transplant: A case report. Exp Clin Transplant 2014;12:165-8.

28. Akhi MT, Bidar Asl S, Pirzadeh T, Naghili B, Yeganeh F, Memar Y, et al. Antibiotic sensitivity of clostridium perfringens isolated from faeces in Tabriz, Iran. Jundishapur J Microbiol 2015;8:e20863.

29. Gamboa-Coronado Mdel M, Mau-Inchaustegui S, RodríguezCavallini E. Molecular characterization and antimicrobial resistance of clostridium perfringens isolates of different origins from Costa Rica. Rev Biol Trop 2011;59:1479-85.

How to cite this article:

Hmood AM, Al-Shukri MSM, Al-Charrakh AH. Molecular detection and antimicrobial resistance of Clostridium perfringens isolated from diabetic patients and bullet wounds. J App Biol Biotech. 2019;7(01):54-59.

DOI: $10.7324 / J A B B .2019 .70110$ 